\title{
Communication Strategies for Strip-Like Topologies in Ad-Hoc Wireless Networks
}

\author{
Daniele De Caneva, Pier Luca Montessoro and Davide Pierattoni \\ University of Udine \\ Italy
}

\section{Introduction}

Many routing protocols have been designed for wireless sensor networks considering nodes that operate in a mesh topology. For specific application scenarios, however, a mesh topology may not be appropriate or simply not corresponding to the natural node deployment. Bridge (Kim et al., 2007) or pipeline (Jawhar et al., 2007) monitoring applications are examples where the position of sensor nodes is predetermined by the physical structure and application requirements. In this applications, where is clearly present a privileged dimension, it is quite natural to take advantage of it. Similar consideration can be made in more dynamic applications like the one of vehicular communication since the network can be approximated to have a linear topology without loss of accuracy.

This chapter will go through a description of the strategies developed so far to handle the problem of communication in strip-like topologies. For this specific problem several studies can be found in literature. Few research directions can be outlined: strip oriented routing, physical device design and specific MAC protocols. In the following four approaches are presented in order to describe how each direction can be investigated. The first two are related to the network layer of ISO/OSI protocol stack, the third one proposes use of devices with directional antennas while the fourth one designs a MAC protocol based on synchronous transmit-receive patterns. These approaches are somewhat complementary, each better suited for different scenarios.

\section{Routing Layer Strategies}

\subsection{MERR}

MERR (Minimum Energy Relay Routing) is a routing protocol which aims to address the problem of an economical use of power in wireless sensor networks. The goal is to minimize power consumption during communications in order to build networks for long-lasting operations. Its reference scenario is that of networks where sensors are deployed over a linear topology and have to send data to a single control center.

Assuming homogeneous sensor nodes deployed in an arbitrary linear sensor network, MERR permits every node to independently find a route to the base station that 
approximates the optimal routing path. Finding a route means selecting appropriate relays between a sensor and the base station.

The problem of relaying data from nodes to the control center can be approached in two ways. The first is direct transmission, where every node transmits its packets directly to the base station. This approach suffers from important problems: first of all, in an environment with many obstacles or if the distance is too large, successful reception at the base station might not be feasible. Secondarily, with direct transmission, since the effort related with transmission increases as a power function of the distance, nodes far away from the base station will suffer greater power consumption and thus exhaust quickly their battery. From this considerations becomes clear that direct transmission is ideal only for scenarios where nodes are close to the base station or when the energy required for reception is large. In that case transmitting data directly to the control center, limits energy dissipation due to reception at the base station (which usually have unlimited power supply).

The second approach consists in taking advantage of the other nodes by using them as routers to forward data packets to the control center. MERR follows this method and in particular states the rules for router choice. MERR authors (Zimmerling et al., 2007) take distance from the MTE policy of routing where routers are chosen in order to minimize transmit energy. Minimizing transmit energy means choosing the nearest neighbor as router, with the evident drawback that a huge amount of energy is wasted if nodes are close to each other or the energy required for reception is high. MERR tries to respond to the question concerning which node must be chosen as router in order to obtain an energy efficient network. Zimmerling et al. based their work on that presented by Bhardwaj et al. (2001) where it is demonstrated that the optimal number of hops to reach a base station situated at a distance $\mathrm{D}$ is always:

$$
\mathrm{K}_{\mathrm{opt}}=\left\lfloor\frac{\mathrm{D}}{\mathrm{d}_{\text {char }}}\right\rfloor \text { or } \quad \mathrm{K}_{\mathrm{opt}}=\left\lceil\frac{\mathrm{D}}{\mathrm{d}_{\text {char }}}\right\rceil
$$

where $d_{\text {char }}$ is the characteristic distance, given by

$$
\mathrm{d}_{\text {char }}=\sqrt[n]{\frac{\alpha_{1}}{\alpha_{2}(\mathrm{n}-1)}}
$$

where $a_{1}, a_{2}$ and $\varepsilon$ are parameters related to node's transceiver circuitry such that the power consumption involved in relaying $r$ bit per second to a distance $d$ meters onward (assuming a path loss of $1 / \mathrm{d}^{\mathrm{n}}$ ) is

$$
\mathrm{P}_{\text {relay }}(\mathrm{d})=\left(\alpha_{1}+\alpha_{2} \mathrm{~d}^{\mathrm{n}}\right) \mathrm{r}
$$

These results show that best performances are reached when packets perform $\left(\mathrm{K}_{\mathrm{opt}}-1\right)$ relays by means of nodes equally spaced in intervals of $\mathrm{D} / \mathrm{K}_{\text {opt }}$.

Based on these assumptions, MERR states that every node should decide independently which will be its relay node. The choice is made seeking the down-stream node within the maximum transmission range whose distance is closest to the characteristic distance. After this decision is made by every node in the network, transmission power is independently reduced to the lowest possible level so that the radio signal can be received by the next-hop node without any errors. During normal functioning, a node will transmit data always to 
the chosen relay node, regardless that this data comes from internal sensors or from another node.
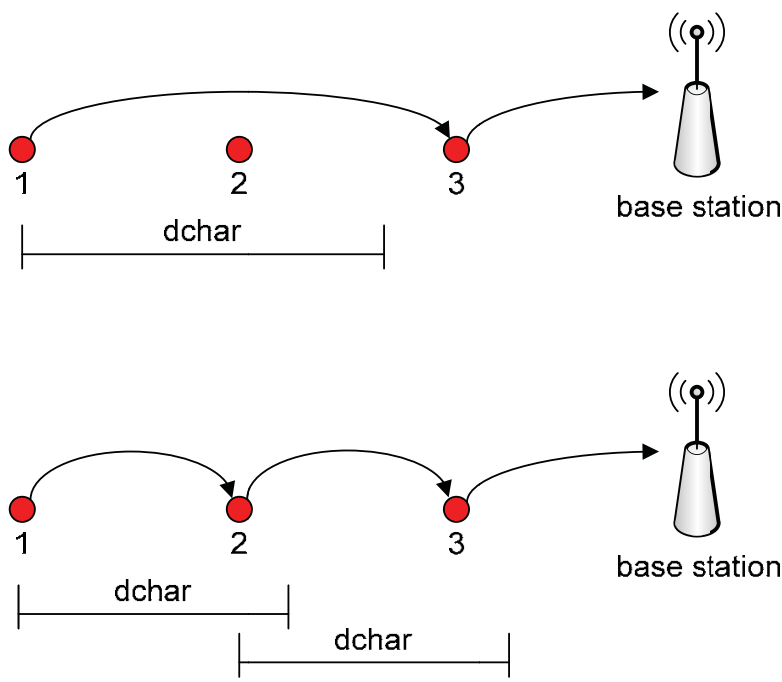

Fig. 1. Characteristic distance influences packets routing path.

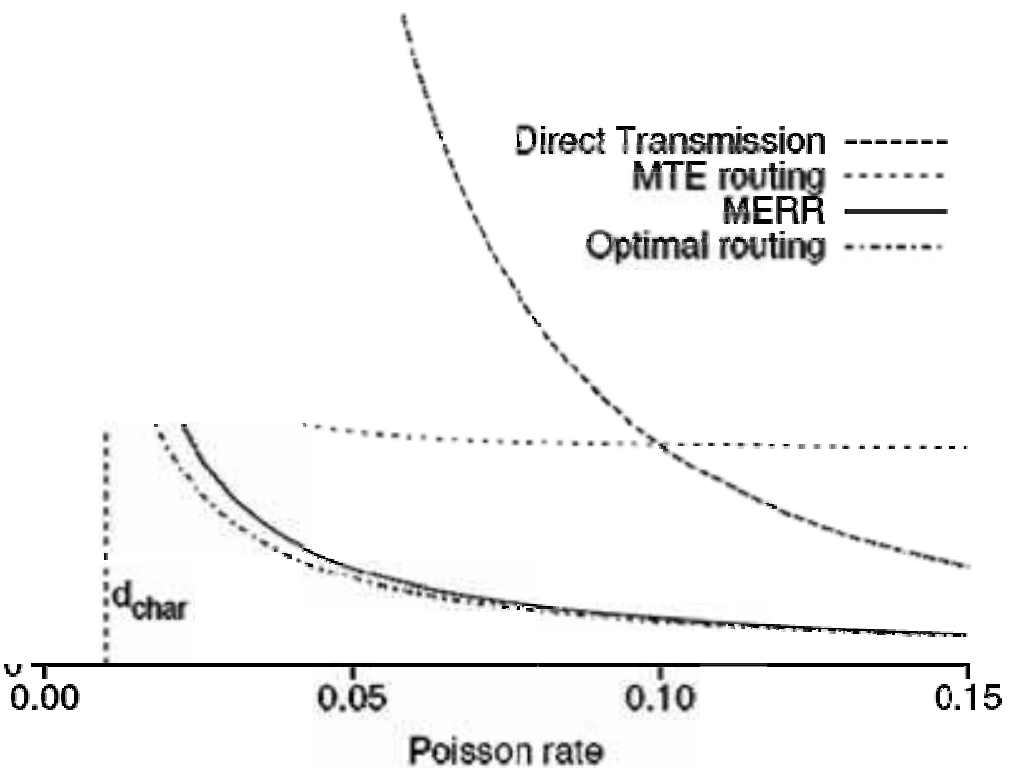

Fig. 2. Expected power consumption depending on Poisson rate $\lambda$ for a constant number of sensors $(n=100)$ and path loss exponent 2 . 
In order to chose its own relay node, every sensor must know the characteristic distance (which is the same for all node if they are of the same kind) and the distance of all its neighbors (which can be manually measured during deployment or estimated using one of the methods present in literature such as Received Signal Strength or Time of Arrival).

Zimmerling et al. offer a comparison in terms of expected power consumption between MERR, optimal transmission, MTE and direct routing. For the sake of generality, the comparison is made using a one-dimensional homogeneous Poisson process with constant rate $\lambda$ to model the distribution of nodes. The comparison, drown from a stochastic analysis made by the authors of MERR, clearly shows that energy consumption of MERR is always upper bounded by that of MTE. In particular MERR require less energy if the mean distance between nodes is lower than the characteristic distance.

\subsection{Load Balanced Short Path Routing}

Although not directly focused on strip-like topologies, the work presented by Gao et al. (2006) is worth mentioning because it covers the special case of a network where nodes are located in a narrow strip with width at most $\sqrt{3} / 2 \approx 0.86$ times the communication range of each node.

Gao et al. tried to harness the main problem afflicting wireless networks, i.e. energy constraints. In particularly they focused on routing layer pointing out that, by minimizing path length, shortest path routing approaches minimize latency and overall energy consumption but may ignore fairness. In fact a protocol that searches the shortest path to route packets, will tend to abuse of some set of hops not exploiting all network resources. This behavior will quickly drain the batteries of involved nodes, causing the creation of holes within the network.

On the other hand load balanced routing strategies aim to use all available network resources in order to even the load, not regarding about communication performances.

Gao et al. in their work combined greedy strategies used to minimize path length and those used to evenly distribute load with the aim to achieve good performances in both metrics of latency and load balance. The problem of finding the most balanced routes is NP-hard even for a simple network and that is why Gao et al. firstly concentrated their efforts on a particular topology. The basic idea of their work is to maintain for each node a set of edges, called bridges, that are guaranteed to make substantial progress. In addiction their paper shows that, when a node has many neighbors, by distributing a collection of binary search trees on the nodes, memory needed on each node and routing/update cost can be reduced significantly.

The routing algorithm relies on two assumptions. The first is that each node knows its location, the second is that the rough location of the destination is known such that the source node knows whether it should send the packet toward its left or right.

For each node $\mathrm{p}, \mathrm{bc}$ is a right (left) bridge if $\mathrm{b}$ and $\mathrm{c}$ are a couple of nodes visible to each other such that $b$ is directly reachable by $p$, while $c$ lies outside the communication range in a position that is right (left) to that of $\mathrm{p}$ (see Fig. 3). The load associated to the bridge is defined as maximum between the loads of $b$ and $c$.

The routing is organized as follows: when $\mathrm{p}$ receives a packet, it first checks if the destination is a direct neighbor. In that case, it sends the packet to the destination. Otherwise, $\mathrm{p}$ chooses the lightest bridge, say bc, that forward the packet toward the 
destination. Then $\mathrm{p}$ send the packet to $\mathrm{b}$, where the process is repeated and so on till the destination is reached.

Gao et al provided a thorough demonstration that the algorithm works under the condition that strip width is equal or minor times the communication range of nodes. Additionally they presented simulation results over different network and traffic conditions.

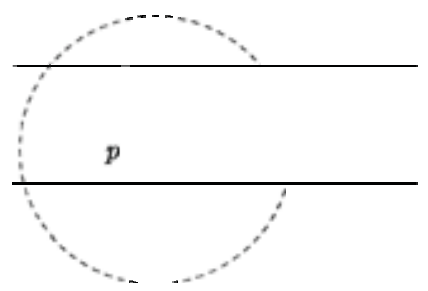

Fig. 3. Communication over a bridge.

\section{MAC Layer Strategies}

\subsection{DiS-MAC}

DiS-MAC (Directional Scheduled MAC) has been developed for wireless sensor networks that show a linear topology. It bases its functioning on a particular use of antennas. The premises that lead to this protocol is that directional or smart antennas have the potential to offer increased spatial reuse, longer communication ranges and the ability to point the radio beam toward a desired direction, properties that if properly exploited could potentially lessen the problem of interferences between nodes. Authors of DiS-MAC (Karveli et al., 2008) pointed out that current advances in antenna miniaturization techniques will open the doors of wireless sensor networks world to this kind of radiating systems.

The reference scenarios is that of highway and roadside monitoring sensors networks. Since roadsides and highways can be approximated to have a linear topology without loss of accuracy, Karveli et al. concentrated their effort on a sensor network deployed in such topology and consisting of $\mathrm{N}$ static nodes generating data packets of equal length with an arbitrary traffic rate. Every node is equipped with a directional antenna that can concentrate the main-beam to a particular direction and presents a some low gain side-lobes in other directions.

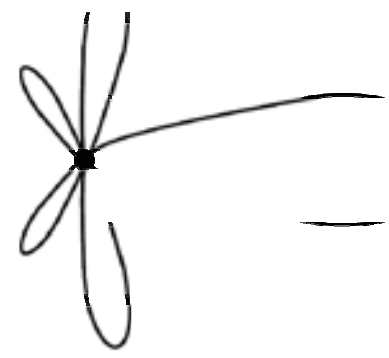

Fig. 4. Model of the antenna system radiation pattern. 
Figure 4 shows the model for the radiation pattern used to develop the protocol. Other assumption for this protocol are that nodes are synchronized and that the traffic flows only in one direction.

Network synchronization permits to divide channel access in two phases of equal length. In the first phase every node occupying a odd position $(2 n-1)$ directs its radiation beam in order to point to the subsequent node and then transmits its data. In this phase nodes occupying a even position ( $2 n$ ) switch their transceiver in reception mode. During the second phase roles are inverted: this time even nodes transmit data to their next node, while odd nodes perform reception. The alternation of phase I and phase II will continue indefinitely.

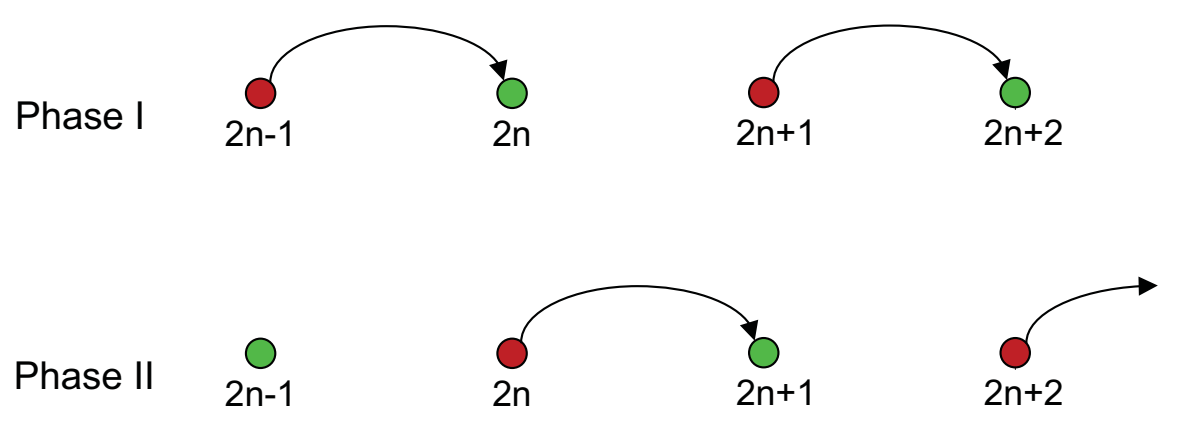

Fig. 5. Two phases scheduling.

This scheduled system provides a great efficiency, since it remove the possibility of collisions and the hidden terminal problem. In fact, since there is no contention, there is no need of control packets and thus it doesn't suffer from the overhead produced by them. This neatly configured system deterministically reaches a channel utilization equal to $1 / 2$. This is quite impressive since in literature ( $\mathrm{Li}$ et al, 2001) it is shown (both by simulations and experiments) that the capacity of a IEEE 802.11 network deployed in chain topology is limited to only 1/7. Additionally, thanks to the absence of channel contention, per hop latency, i.e. the time spent from packet generation at one node to its reception at the next node, is minimized and can be approximated by the duration of two phases.

Moreover the protocol is intrinsically robust because it limits interference between nodes, in fact when a node transmits, the first downstream node that can eventually suffer from this transmission is 3 hops ahead. Thus even considering the common assumption that the interference radius is twice the nominal transmission one, as shown in Fig. 6, DiS-MAC grants the avoidance of intra-network interference problems.

Authors of DiS-MAC outlined two extensions for their protocol. The first is a minor one, which states that if a node has no packet to transmit, it can enter into a sleeping mode. If another node have to transmit a packet to this sleeping node, it have to generate a short wake up radio signal in order to warn about the imminent transmission.

The second enhancement consist in the introduction of ACK packets to confirm that the transmitted packet has reached its destination without errors. Thanks to the contention-free nature of DiS-MAC, the repeated absence of ACK reception can be used as a marker of node failure. In this case, Karveli et al. have thought a strategy to react to the topology change. If node $2 n$ fails, neither node $2 n-1$ nor node $2 n+1$ will receive its packets (the first one will 
receive no ACK packet, while the second will receive no data packet). After a predefined counter expires, node and node will consider their neighbor failed and will start a recovery procedure. The first will extend its transmission range in order to reach the second one and then it will send a phase change request, which will propagated to all subsequent nodes. Phase change request is made through a special control packet and warns a node that its position into the chain is changed (e.g. node is become node

) and that it have to modify its behavior according to new topology. To avoid false failure detection, this protocol extension requires the transmission of periodical keep-alive packets.
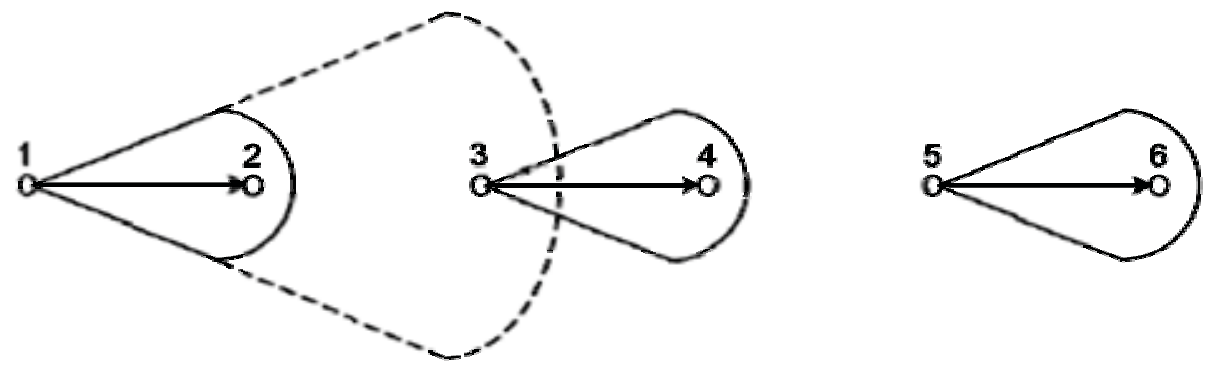

Fig. 6. Interference radius (dashed line) and transmit radius (solid line).

\subsection{WiWi}

The purpose of WiWi (De Caneva et al., 2008) is to emulate a wired link by means of an ad hoc network constituted by nodes distributed along a strip. The purpose of this wired link virtualization is that to handle scenarios where a single hop wireless link is not feasible and a wired link is not practical. An example could be given by a speleologist going deep down into the bowels of the earth, which can deploy the wireless network while it goes further with the exploration in order to maintain a communication channel with the outside world. Other examples can be found in all those situation where a multi-hop link is required. Moreover WiWi can be successfully used in monitoring applications.

Results presented in (Min \& Chandrakasan, 2003) regarding power consumption over multihop networks demonstrated that the number of hops used to route a packet from source to destination should not be too high. In fact in such situation the portion of power consumption which is independent by the transmission distance becames predominant, thus causing energy saving obtained by shorter transmission hops to be nullified. Moreover, the higher the hops number, the higher the latency. Nevertheless, the coverage range is limited by nodes' architectural characteristics and this define a lower bound for the hops number. This considerations led WiWi developers to choose a non-uniform node displacement and thus a cluster chain topology.

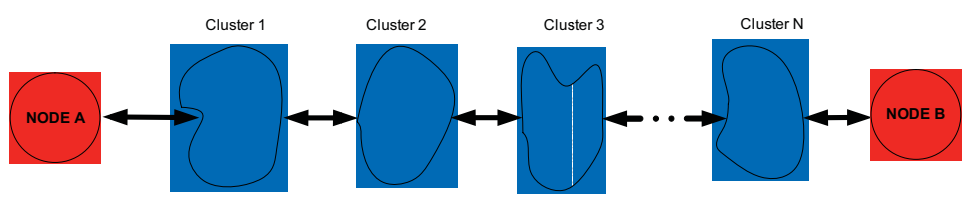

Fig. 7. WiWi topology. 
De Caneva et al. made no assumptions over node deployment within the clusters, but full inter-cluster graph connection as well as complete radio coverage between nodes belonging to adjacent clusters.

WiWi protocol follows a synchronous full-duplex communication with fixed-side packets where clusters act as single nodes. In particular there exists two data stream which proceed along the chain in two different manners, depending on the direction. The first is a downward stream that relays packets from the head of the strip to the tail (gray packets in Fig. 8). This stream, which is responsible of maintaining network synchronization, follows a staggered pattern, i.e. a cluster sends a packet to the next cluster, which in turn immediately forwards it further down along the chain. This stream shows a latency equal to $L_{\text {downlink }}=$ hops $T_{s}$, where $T_{s}$ is the length of a time slot. The throughput associated with this stream can be expressed as the ratio between the number of bits forming a packet and the time interleaving two consecutive downstream transmissions (i.e. $\sigma T_{S}$, where $\sigma$ is the number of slots by which spaces two consecutive transmissions).

The opposite stream follows the same principle of passing messages along the cluster chain, but between the reception of the packet and its forwarding, the cluster waits four time slots in order not to collide with the downward (Fig. 8 shows in different colors the steps taken by different upward packets). The latency affecting the upward stream is $\sigma-1$ times the one of the downward, while the throughput is the same.

WiWi protocol is based on datagram transmission, in fact does not provide ACK packets to guarantee the correct packet exchange. Authors of WiWi point out that, if needed, the use of error correction codes could be introduced as well as acknowledgement mechanisms at higher level protocols.

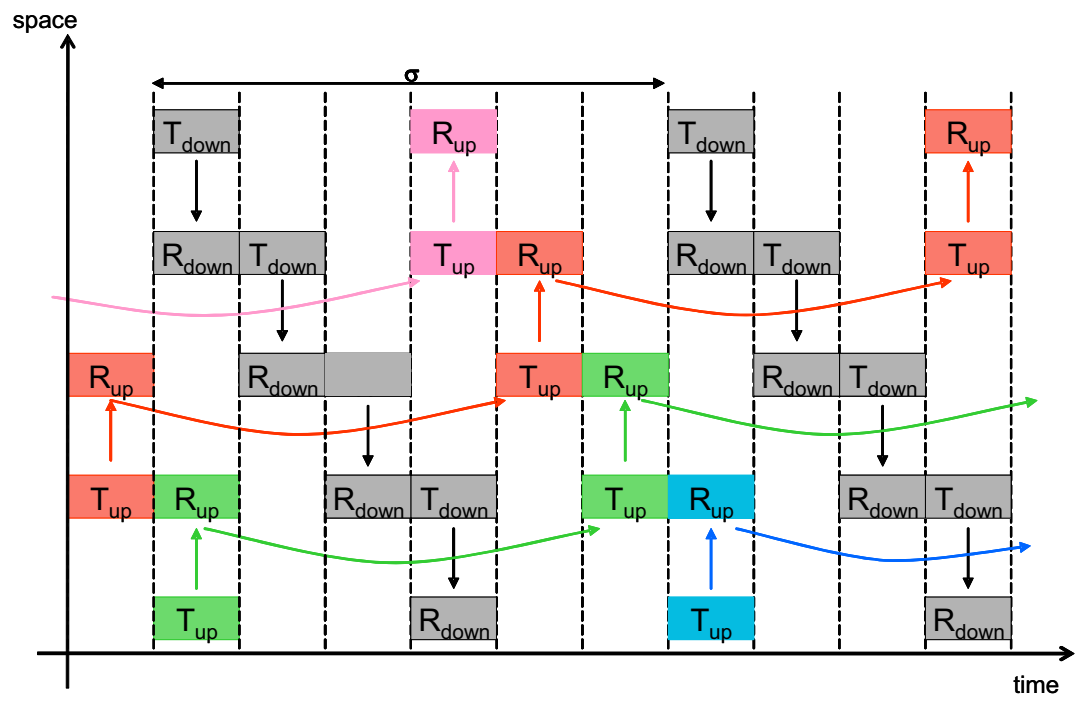

Fig. 8. Bidirectional, staggered transmission with symmetric throughput and asymmetric latency over a WiWi link. 
As previously mentioned WiWi clusters act as a single node. This is done in order to reach redundancy as well as load balance. In fact WiWi requires that each cluster independently organize itself by ordering its nodes. By ordering a node belonging to the cluster can be elected as node on duty, i.e. the node that have to perform the packet relaying operations that compete to the cluster. The other nodes act as backup nodes. Operatively, during the reception slot every node of the cluster receives and stores the packet arriving from the previous cluster in the chain. In the subsequent transmission slot, the node on duty forwards the packet, while at the same time all backup nodes perform a sensing of the wireless channel. If the backup nodes perceive the loss of the duty node, they react autonomously by redefining their order within the cluster. This way the backup node, which would have its turn next to the current duty node, takes the role of forwarding the packet. The remaining backup nodes perform the sensing again in order to be sure that a backup node has reacted and the forwarding has occurred (Fig. 9). WiWi protocol grants an immediate redundancy equal to the number of backup nodes, which is the total number of active nodes in a cluster minus one. This means that the slot time upon which WiWi is based must have a duration capable to conserve this redundancy mechanism, which lead to a minor loss in throughput and latency performances. In the packet header could be inserted a notification flag to inform subsequent clusters of the failure event.

Clearly the node on duty is burdened with a higher power consumption, that is why nodes in turn cover this role following a round robin schedule. Additionally the scheduling of the duty evenly shares the load among cluster nodes extending the network lifetime and opening the door to the use of energy scavenging techniques.

The bandwidth unused by the redundancy mechanism, in normal conditions could be periodically exploited to reorganize each cluster on the run, in order to take care of the postdeployed nodes, if any.

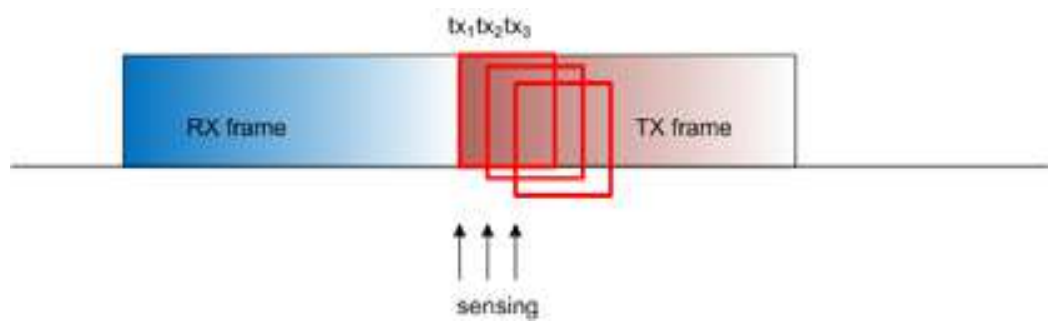

Fig. 9. Cluster redundancy management.

\section{Conclusion}

In this chapter were presented four algorithms whose aim is to manage packet relaying within an ad-hoc wireless network formed by nodes deployed over a strip. This algorithms are not exactly competing, instead they are focused on somewhat different scenarios which are related to different applications and hardware capabilities. In a field like the one of wireless sensor networks, where hardware constraints and application needs arise extremely challenging problems, taking every possible advantage is crucial. From this point of view it is clear that research have to develop new algorithms and protocols which exploit 
network topology. Algorithms for linear and strip topologies represent the first steps toward this new trend of topology-oriented protocols.

\section{References}

Bhardwaj, M.; Garnett, T., Chandrakasan, A., "Upper bounds on the lifetime of sensor networks", Proceedings of IEEE International Conference on Communications (ICC 2001), pp. 785-790, Jun. 2001.

De Caneva, D.; Montessoro, P.L.; Pierattoni, D., "WiWi: Deterministic and Fault Tolerant Wireless Communication Over a Strip of Pervasive Devices", Proceedings of Wireless Communications, Networking and Mobile Computing, 2008. WiCOM '08. 4th International Conference on, pp.1-5, 12-14 Oct. 2008.

Gao, J.; Zhang. L., "Load-balanced short-path routing in wireless networks", Parallel and Distributed Systems, IEEE Transactions on , vol.17, no.4, pp. 377-388, April 2006

Karveli, T.; Voulgaris, K.; Ghavami, M.; Aghvami, A.H., "A Collision-Free Scheduling Scheme for Sensor Networks Arranged in Linear Topologies and Using Directional Antennas", Proceedings of Sensor Technologies and Applications, 2008. SENSORCOMM '08. Second International Conference on, pp.18 - 22, 25-31 August 2008.

Kim, S.; Pakzad, S.; Culler, D.; Demmel, J.; Fenves, G.; Glaser, S; Turon, M, Health "Monitoring of Civil Infrastructures Using Wireless Sensor Networks", Proceedings of Information Processing in Sensor Networks, 2007. IPSN 2007. 6th International Symposium on, pp. 254-263, 25-27 April 2007.

Jawhar, I.; Mohamed, N.; Shuaib, K., "A framework for pipeline infrastructure monitoring using wireless sensor networks", Proceedings of Wireless Telecommunications Symposium, 2007. WTS 2007, pp. 1-7, 26-28 April 2007.

Li, J.; Blake C.; De Couto D.S.J.; Imm Lee, H.; Morris, R., "Capacity of Ad Hoc Wireless Networks", Proceedings of Mobile Computing and Networking, $7^{\text {th }}$ ACM International Conference on, pp. 61-69, July 2001.

Min, R. \& Chandrakasan, A., "Top Five Myths about the Energy Consumption of Wireless Communication", ACM SIGMOBILE Mobile Computing and Communications Review, Vol. 1, No. 2, 2003.

Zimmerling, M.; Dargie, W.; Reason, J.M., "Energy-Efficient Routing in Linear Wireless Sensor Networks", Proceedings of Mobile Adhoc and Sensor Systems, 2007. MASS 2007. IEEE Internatonal Conference on, pp. 1-3, 8-11 October 2007. 


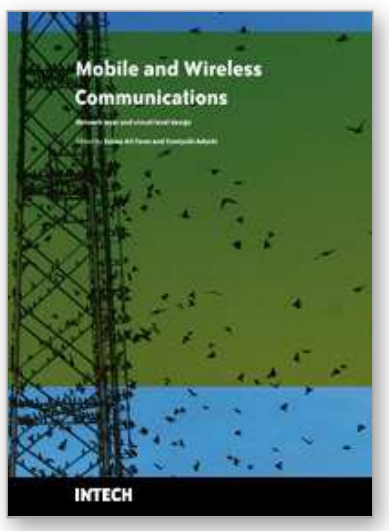

\section{Mobile and Wireless Communications Network Layer and Circuit Level Design}

Edited by Salma Ait Fares and Fumiyuki Adachi

ISBN 978-953-307-042-1

Hard cover, 404 pages

Publisher InTech

Published online 01, January, 2010

Published in print edition January, 2010

Mobile and wireless communications applications have a clear impact on improving the humanity wellbeing. From cell phones to wireless internet to home and office devices, most of the applications are converted from wired into wireless communication. Smart and advanced wireless communication environments represent the future technology and evolutionary development step in homes, hospitals, industrial, vehicular and transportation systems. A very appealing research area in these environments has been the wireless ad hoc, sensor and mesh networks. These networks rely on ultra low powered processing nodes that sense surrounding environment temperature, pressure, humidity, motion or chemical hazards, etc. Moreover, the radio frequency (RF) transceiver nodes of such networks require the design of transmitter and receiver equipped with high performance building blocks including antennas, power and low noise amplifiers, mixers and voltage controlled oscillators. Nowadays, the researchers are facing several challenges to design such building blocks while complying with ultra low power consumption, small area and high performance constraints. CMOS technology represents an excellent candidate to facilitate the integration of the whole transceiver on a single chip. However, several challenges have to be tackled while designing and using nanoscale $\mathrm{CMOS}$ technologies and require innovative idea from researchers and circuits designers. While major researchers and applications have been focusing on RF wireless communication, optical wireless communication based system has started to draw some attention from researchers for a terrestrial system as well as for aerial and satellite terminals. This renewed interested in optical wireless communications is driven by several advantages such as no licensing requirements policy, no RF radiation hazards, and no need to dig up roads besides its large bandwidth and low power consumption. This second part of the book, Mobile and Wireless Communications: Key Technologies and Future Applications, covers the recent development in ad hoc and sensor networks, the implementation of state of the art of wireless transceivers building blocks and recent development on optical wireless communication systems. We hope that this book will be useful for students, researchers and practitioners in their research studies.

\section{How to reference}

In order to correctly reference this scholarly work, feel free to copy and paste the following:

Daniele De Caneva, Pier Luca Montessoro and Davide Pierattoni (2010). Communication Strategies for StripLike Topologies in Ad-Hoc Wireless Networks, Mobile and Wireless Communications Network Layer and Circuit Level Design, Salma Ait Fares and Fumiyuki Adachi (Ed.), ISBN: 978-953-307-042-1, InTech, Available from: http://www.intechopen.com/books/mobile-and-wireless-communications-network-layer-and-circuit-leveldesign/communication-strategies-for-strip-like-topologies-in-ad-hoc-wireless-networks 
open science | open minds

\section{InTech Europe}

University Campus STeP Ri

Slavka Krautzeka 83/A

51000 Rijeka, Croatia

Phone: +385 (51) 770447

Fax: +385 (51) 686166

www.intechopen.com

\section{InTech China}

Unit 405, Office Block, Hotel Equatorial Shanghai

No.65, Yan An Road (West), Shanghai, 200040, China

中国上海市延安西路65号上海国际贵都大饭店办公楼 405 单元

Phone: +86-21-62489820

Fax: +86-21-62489821 
(C) 2010 The Author(s). Licensee IntechOpen. This chapter is distributed under the terms of the Creative Commons Attribution-NonCommercial-ShareAlike-3.0 License, which permits use, distribution and reproduction for non-commercial purposes, provided the original is properly cited and derivative works building on this content are distributed under the same license. 